\title{
Effect of pERK2 on extracellular matrix turnover of the fibrotic joint capsule in a post-traumatic joint contracture model
}

\author{
YANGBAI SUN*, FENGFENG LI* and CUNYI FAN \\ Department of Orthopedics, Shanghai Jiaotong University Affiliated Sixth People's Hospital, \\ Shanghai 200233, P.R. China
}

Received March 26, 2015; Accepted November 5, 2015

DOI: $10.3892 /$ etm.2015.2948

\begin{abstract}
Lentivirus (LV)-mediated extracellular signal-regulated kinase (ERK)2 small interfering RNA (siRNA) has previously been demonstrated to reduce post-traumatic joint contractures: In the present study, the effect of ERK2 siRNA on extracellular matrix turnover within fibrotic joint capsules in post-traumatic joint contractures was examined. Rats were randomly assigned to one of three groups as follows: The non-operated control (CON), operated contracture (ORC) and contracture-treatment (CNT) groups. Representative post-traumatic joint contracture was created through 8 weeks of immobilization following intra-articular injury. In the CNT group, LV-mediated ERK2 siRNA was injected into the model knee at days 3 and 7 after surgery. The posterior joint capsule was examined by western blotting, histology and immunohistochemistry to evaluate alterations in ERK2, phosphorylated (p)ERK2, total collagen, collagen I, collagen III, matrix metalloproteinase (MMP)-1, MMP-13 and tissue inhibitor of metalloproteinase (TIMP)-13. In the ORC group, pERK2 was elevated and total collagen, collagen I, MMP-1 and MMP-13 were significantly increased ( $\mathrm{P}<0.01$ vs. CON group); however, these were significantly decreased in the CNT group, and pERK2 was downregulated $(\mathrm{P}<0.01$ vs. ORC group). Collagen III and TIMP-13 were markedly decreased in the ORC group $(\mathrm{P}<0.01$ vs. CON group), but elevated in the CNT group ( $\mathrm{P}<0.01$ vs. ORC group). The present res4ults demonstrate unique pathological changes of the fibrotic joint capsule that are responsible for joint contracture following traumatic injury, and reveal that extracellular matrix turnover can be affected by pERK2.
\end{abstract}

Correspondence to: Professor Cunyi Fan, Department of Orthopedics, Shanghai Jiaotong University Affiliated Sixth People's Hospital, 600 Yishan Road, Shanghai 200233, P.R. China

E-mail: fancunyi888@hotmail.com

*Contributed equally

Key words: joint contracture, collagen, matrix metalloproteinase-1, matrix metalloproteinase-13, tissue inhibitor of metalloproteinase-13

\section{Introduction}

Joint contracture is a major complication following elbow trauma that restricts joint motion, and additional surgical procedures are often required for the treatment of severe joint contracture. In the anatomical context of a contracted joint, the joint capsule is regarded as the critical motion-limiting structure (1). In patients with chronic elbow contractures following trauma, the fibrotic joint capsule becomes markedly thickened and disorganized compared with normal elbows $(2,3)$. Cellular, matrix, and growth factor components of the joint capsule are changed during the formation of fibrotic joint capsules in a post-traumatic contracture model (4). Multiple scientific groups are, therefore, investigating novel strategies to reduce joint capsule fibrosis in order to prevent joint contracture.

Prior studies have reported that ketotifen, a mast cell stabilizer, inhibits joint capsule fibrosis in a joint contracture model following trauma $(5,6)$. The formation of joint contractures may also be inhibited by the intra-articular injection of lentivirus (LV)-mediated extracellular signal-regulated kinase 2 (ERK2) small interfering RNA (siRNA); myofibroblast hyperplasia and its subsequent reduction also appear to be affected by the downregulation of pERK2 (7).

In the present study, the effects of LV-mediated ERK2 siRNA treatment of the fibrotic joint capsule of a contracted joint were, therefore, investigated; specifically, the effects upon extracellular matrix components, including total collagen, collagen I, collagen III, matrix metalloproteinase (MMP)-1, MMP-13 and tissue inhibitor of metalloproteinase (TIMP)-13 were evaluated.

\section{Materials and methods}

Group allocation. All experimental procedures were approved by the Institutional Animal Review Committee of Shanghai Jiaotong University Affiliated Sixth People's Hospital (Shanghai, China). A total of 57 female rats, weighing $0.22-0.28 \mathrm{~kg}$ and aged 3 months, were purchased from the Shanghai SLAC Laboratory Animal Co., Ltd. (Shanghai, China). As previously described (7), the rats were randomly separated into three groups ( $n=19$ in each), as follows: The operated contracture (ORC) group, the contracture-treatment (CNT) group and the non-operated control (CON) group. In the ORC and CNT groups, representative post-traumatic joint 
contracture was developed through 8 weeks of immobilization following surgical intra-articular injury. Rats of the CNT group then received an intra-articular injection of $0.1 \mathrm{ml} \mathrm{LV}$ particles containing ERK2 siRNA at days 3 and 7 after surgery. The rats of the CON group did not receive any surgical or pharmacological intervention.

LV-mediated ERK2 siRNA construction and in vivo bioluminescence detection. The siRNA sequence targeting rat ERK2 (5'-GCACCTCAGCAATGATCAT-3') has previously been reported to efficiently downregulate ERK2 expression in rats (7). Pairs of complementary oligonucleotides containing these sequences were synthesized (Thermo Fisher Scientific, Inc., Waltham, MA, USA ) and cloned into the pshRNA-H1-Luc lentivector (System Biosciences, Mountain View, CA, USA). Next, 293T producer cells were co-transfected with pPACK Packaging Plasmid Mix and the pshRNA-H1-Luc lentivectors containing the shRNA sequences (System Biosciences, Mountain View, CA, USA). Viral supernatants were collected after $48 \mathrm{~h}$, centrifuged at $5,000 \mathrm{xg}$ at $4^{\circ} \mathrm{C}$ for $5 \mathrm{~min}$ to eliminate cell debris and filtered through 0.45 to $1-\mu \mathrm{m}$ polyvinylidene fluoride filters (EMD Millipore, Billerica, MA, USA). The viral titers were determined with serial dilutions of concentrated LV. As previously described (7), the luciferase expression and distribution of the rats in the CNT group were measured using a Xenogen IVIS 50 Bioluminescence System (PerkinElmer, Inc., Waltham, MA, USA) 2 and 8 weeks after surgery. For a basis of comparison, the rats in the ORC group were also imaged using the with Xenogen IVIS 50 Bioluminescence System.

Joint interventions. As previously described (7), the rats were placed in a supine position for surgery under aseptic conditions; general anesthesia was administered via inhalation, using $2-3 \%$ isoflurane and oxygen. Following a midline skin incision in the right knee joint, a lateral parapatellar arthrotomy was performed. The patella was moved medially and the knee joint was flexed to expose the femoral condyles. Two $1.5 \times 1.5-\mathrm{mm}$ cortical windows were created from the non-articulating cartilaginous regions of the medial and lateral femoral condyles using a $1.5-\mathrm{mm}$ drill bit. The anterior and posterior cruciate ligaments were then incised, and the knee was overextended to $45^{\circ}$ to disrupt the posterior joint capsule. The right knee was immobilized at flexion of $140^{\circ}$ with surgical sutures. The patellofemoral joint was appropriately reduced prior to incision closure. Following joint interventions, the rats were permitted unrestricted movement in their cages (10 rats to a cage of $0.1 \mathrm{~m}^{3}$ ).

Western blotting. A total of 15 rats ( $\mathrm{n}=5$ per group) were sacrificed using an overdose of pentobarbital sodium (Sumimoto Dainippon Pharma Co., Ltd., Osaka, Japan) at 2 and 8 weeks, respectively. Posterior joint capsules were then gathered and lysed with lysis buffer. The cell lysates were centrifuged at $13,000 \mathrm{x} g$ for $15 \mathrm{~min}$ at room temperature, and the supernatants collected were used for western blotting. Equal amounts of protein were separated on a $12 \%$ sodium dodecyl sulfate gel using polyacrylamide gel electrophoresis and electrotransferred to nitrocellulose membranes (EMD Millipore). The membranes were blocked with $5 \%$ skimmed milk, and incubated with antibodies against glyceraldehyde 3-phosphate dehydrogenase, ERK2 and pERK (Cell Signaling Technology Inc., Danvers, MA, USA) for $1 \mathrm{~h}$ at room temperature. Following a washing step, the membranes were incubated with horseradish peroxidase-conjugated immunoglobin $G$ (Acris Antibodies, GmbH, Herford, Germany) for $1 \mathrm{~h}$ and immunoreactive bands were detected by chemiluminescence (Amersham Biosciences, Freiburg, Germany).

Histological assessment. As previously described (7), 23 rats across the three groups were euthanized using an overdose of pentobarbital sodium (Dainippon Pharmaceutical) 8 weeks after surgery. Posterior capsule tissues of the right knees were immediately removed and the tissues were cut into halves. One half was quickly frozen for subsequent immunohistochemical staining of $\alpha$-smooth muscle actin for use in another study (7). The other half was fixed with formalin, embedded with paraffin and cut into 4-mm thick sections. A number of sections were stained using the Masson trichrome staining method for collagen, as follows: First, the tissue was deparaffinized and hydrated using graded ethanol and xylene solutions, followed by distilled water. The tissue slides were incubated in Weigert iron hematoxylin stain, followed by counterstaining with Biebrich scarlet-acid fuchsin and aniline blue (Sigma-Aldrich, St. Louis, MO, USA), according to the manufacturer's instructions. Masson trichrome staining was used to demonstrate the association of muscle cells with collagen.

Immunohistochemistry. Immunohistochemistry was performed on the remaining sections. Primary rabbit monoclonal antibodies against rat collagen I (1:1,000; ab34710), collagen III (1:1,000; ab7778), MMP-1 (1:40; ab118529), MMP-13 (1:100; ab39012) and TIMP-1 (1:40; ab61224; Abcam, Cambridge, UK) were applied to slides overnight $\left(\sim 18 \mathrm{~h}\right.$ at $\left.4^{\circ} \mathrm{C}\right)$. Following a washing step, all slides were exposed to the secondary goat anti-rabbit antibody, Super Sensitive Rabbit Link (1:1,000; BioGenex, Shanghai, China), for $1 \mathrm{~h}$ at room temperature and washed again. Finally, the sections were stained with 3,3'-diaminobenzidine for $1 \mathrm{~min}$, and counterstained with hematoxylin. The slides were assessed under an optical microscope (DP75; Olympus Corporation, Tokyo, Japan), and the observer was blinded to which specimen was being examined. The immunohistochemical staining intensities of collagen I and III were graded on a scale of 0-3 as follows: No staining, 0; weak staining, 1; moderate staining, 2; and intense staining, 3. A total of 9 randomly-selected sections were then examined for collagen I and III, which were again assessed by the same observer blinded to the conditions.

Statistical analysis. The data were statistically analyzed using a one-way analysis of variance with Student-Newman-Keuls post hoc t-test. Differences were considered to be statistically significant for values of $\mathrm{P}<0.05$. All statistical analyses were conducted using SPSS 11.0 (SPSS Inc., Chicago, IL, USA).

\section{Results}

Among the 57 rats used in this study, 4 animals were excluded from the study due to a failure to thrive, a tibial fracture and 
Table I. Mean staining intensities of total collagen, collagen type I, collagen type III, MMP-1, MMP-13 and TIMP-13.

\begin{tabular}{lccc}
\hline Target & CON $(\mathrm{n}=8)$ & ORC $(\mathrm{n}=8)$ & $\mathrm{CNT}(\mathrm{n}=7)$ \\
\hline Total collagen & $1.9(0.5)$ & $2.8(0.5)$ & $2.0(0.4)$ \\
Collagen type I & $1.9(0.6)$ & $2.7(0.5)$ & $1.9(0.7)$ \\
Collagen type III & $2.7(0.4)$ & $2.1(0.6)$ & $2.8(0.5)$ \\
MMP-1 & $0.8(0.5)$ & $1.3(0.5)$ & $0.9(0.5)$ \\
MMP-13 & $1.2(0.9)$ & $1.7(0.3)$ & $1.3(0.4)$ \\
TIMP-13 & $1.6(0.5)$ & $1.2(0.9)$ & $1.5(0.6)$ \\
\hline
\end{tabular}

Results expressed as mean (standard deviation). Significant differences were observed between the ORC and CON groups, and between the ORC and CNT groups $(\mathrm{P}<0.05)$. CON, non-operated control; ORC, operated contracture; $\mathrm{CNT}$, contracture-treatment; MMP, matrix metalloproteinase; TIMP-13, tissue inhibitor of metalloproteinase-13.
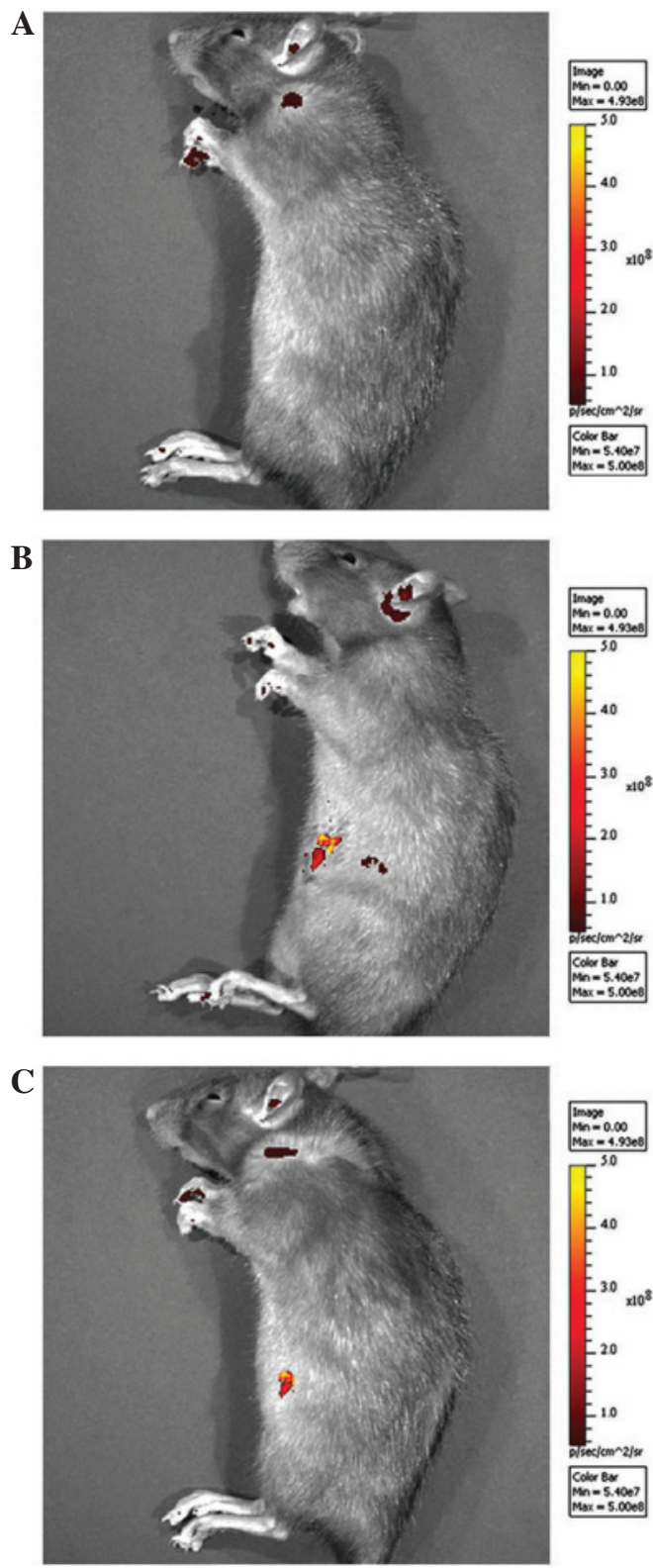

Figure 1. (A) Representative fluorescence image of rats in the operated contracture and (B) contracture-treatment groups at 2 weeks and (C) 8 weeks after surgery.

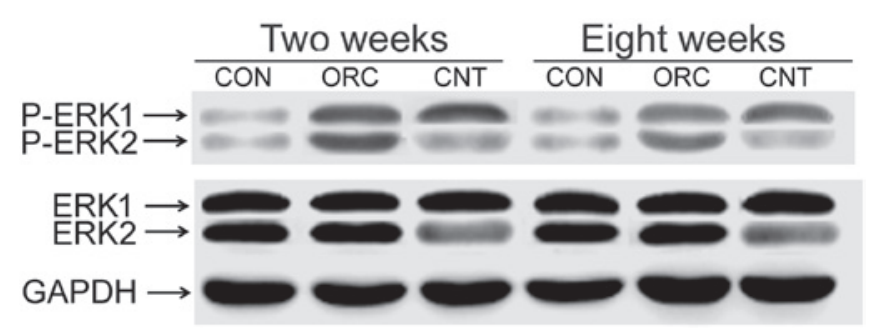

Figure 2. Relative ERK1/2 and pERK1/2 protein levels of the posterior joint capsule in the three groups at 2 and 8 weeks after surgery as determined by western blotting. ERK, extracellular signal-regulated kinase; pERK, phosphorylated ERK; GAPDH, glyceraldehyde 3-phosphate dehydrogenase.

immobilization failure. The other animals appeared healthy, with no signs of impaired wound healing or deep infection.

As previously described (7), fluorescence was detected in the right knee joints of rats of the CNT group 2 weeks after surgery (Fig. 1). At 8 weeks after surgery, luciferase fluorescence did not significantly decrease (Fig. 1), and no fluorescence was detected in the right knee joints of rats of the ORC group (Fig. 1). Western blot analysis demonstrated that pERK2 levels of the posterior joint capsule from rats in the ORC group were significantly increased compared with those in the CON group at 2 and 8 weeks (Fig. 2). However the rate of increase was greater at 2 weeks than at 8 weeks (Fig. 2). The successful injection of LV-mediated ERK2 siRNA into the joints of rats of the CNT group (Fig. 2) specifically downregulated pERK2 levels of the posterior joint capsule by inhibiting ERK2 protein expression levels compared with those in the ORC group at 2 and at 8 weeks (Fig. 2).

The posterior joint capsules of the right knees of 8 rats in the CON group, 8 rats in the ORC group and 7 rats in the CNT group were examined by histology and immunohistochemistry. Under light microscopy, Masson trichrome staining of all control specimens revealed well-organized, parallel, sparse collagen fibers between intervening cells (Fig. 3A). All capsules from the ORC group demonstrated extensive disorganization of the collagen fiber bundle arrangement with a dense structure (Fig. 3B). Similar to the capsule wall structure of the CON group, tissue of the CNT group became sparse, with well-organized collagen fibers (Fig. 3C). Evaluation of the staining intensity of collagen demonstrated significant differences between the ORC and CON groups, and between the ORC and CNT groups $(\mathrm{P}<0.05$; Table I).

Analysis of the staining intensities of collagen I, MMP-1 and MMP-13 demonstrated a marked difference between the contracture and the control capsules (Figs. 4-6). However, the intra-articular delivery of LV-mediated ERK2 siRNA significantly reduced the staining intensities of collagen I, MMP-1 and MMP-13 in capsule tissue of the CNT group compared with the ORC group (Figs. 4-6). However, the staining intensities of collagen III and TIMP-13 were weaker in the ORC group compared with the CON group, but were elevated following LV-mediated ERK2 siRNA treatment of the fibrotic joint capsule in the CNT group (Figs. 7 and 8). Assessment by blinded observers revealed significant differences in staining intensities between the ORC and CON groups, and between the ORC and CNT groups $(\mathrm{P}<0.05$; Table I). 

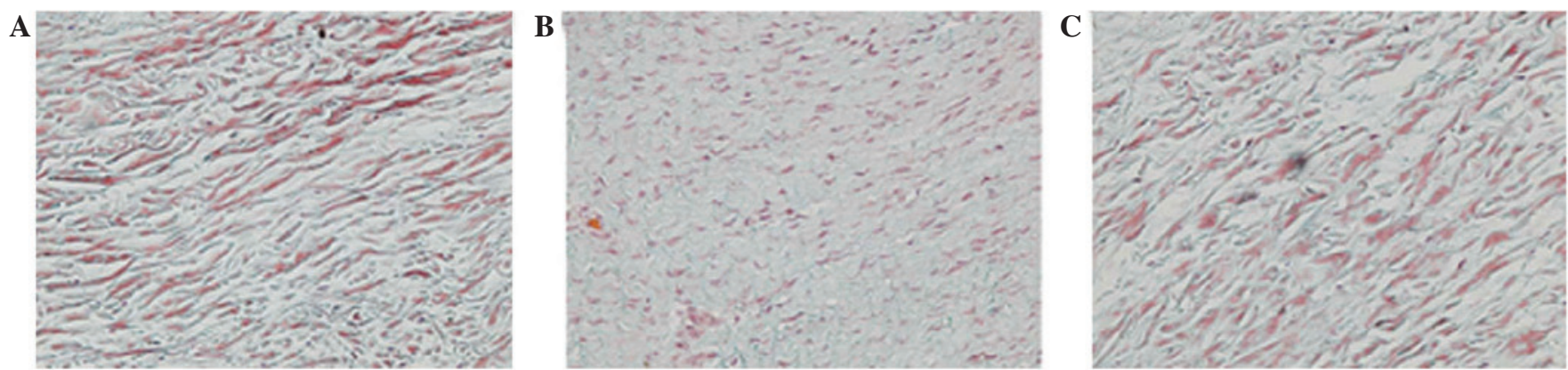

Figure 3. Masson trichrome staining (magnification, $\mathrm{x} 200$ ) in specimens of the (A) CON, (B) ORC and (C) CNT groups. Muscle is indicated in red and collagen in green. Staining intensity of collagen was greater in every specimen of the ORC group compared with every specimen of the CON and CNT groups. CON non-operated control; ORC, operated contracture; CNT, contracture-treatment.
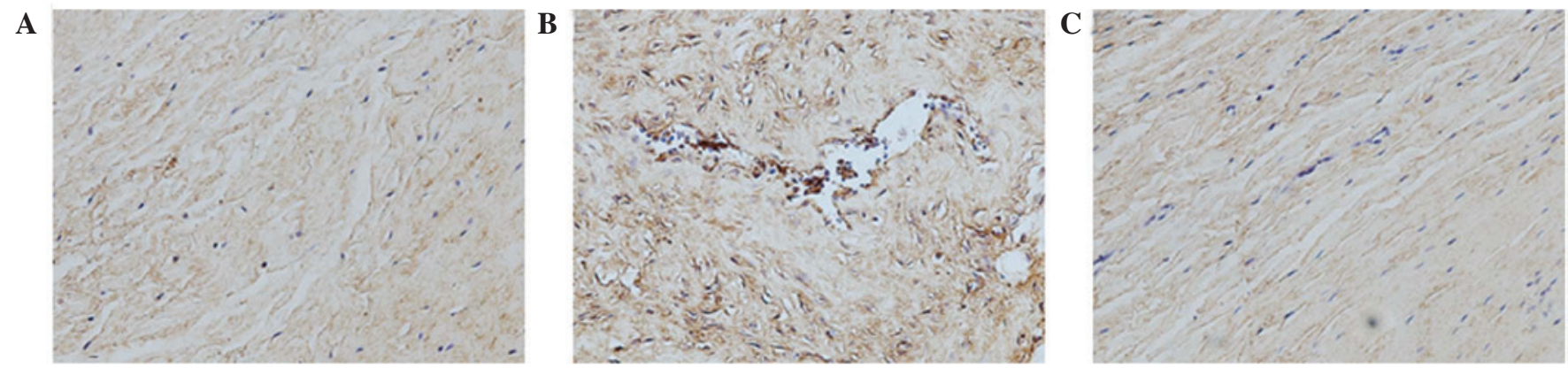

Figure 4. Immunohistochemical staining for collagen I (magnification, $\mathrm{x} 200$ ) in specimens of the (A) CON, (B) ORC and (C) CNT groups. Staining intensity was greater in every specimen of the ORC group compared with every specimen of the CON and CNT groups. CON, non-operated control; ORC, operated contracture; CNT, contracture-treatment.

A

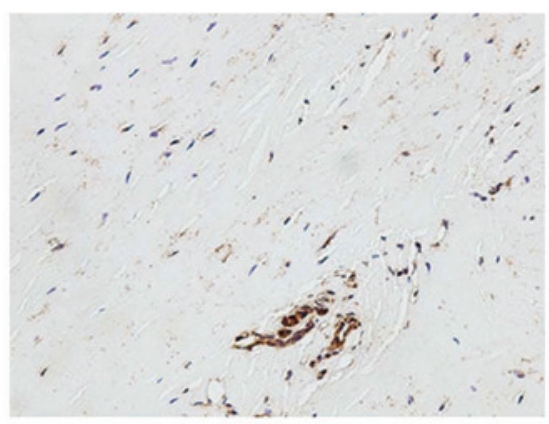

B

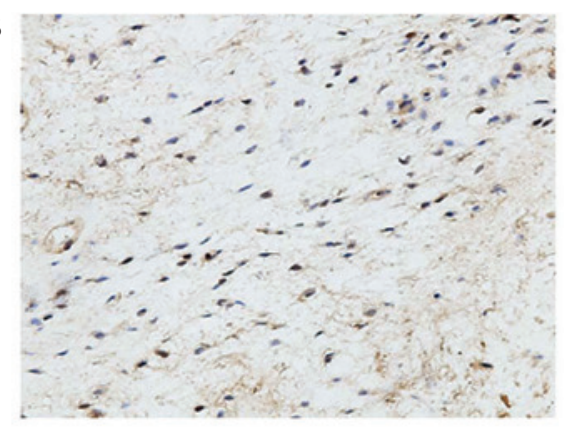

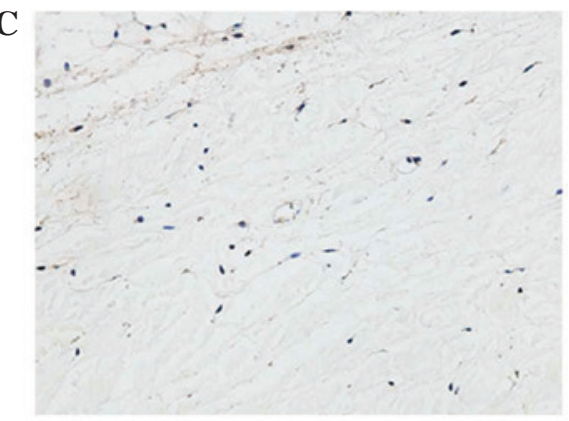

Figure 5. Immunohistochemical staining for matrix metalloproteinase-1 (magnification, x200) in specimens of the (A) CON, (B) ORC and (C) CNT groups . Staining intensity was greater in every specimen of the ORC group compared with every specimen of the CON and CNT groups. CON, non-operated control; ORC, operated contracture; CNT, contracture-treatment.

A

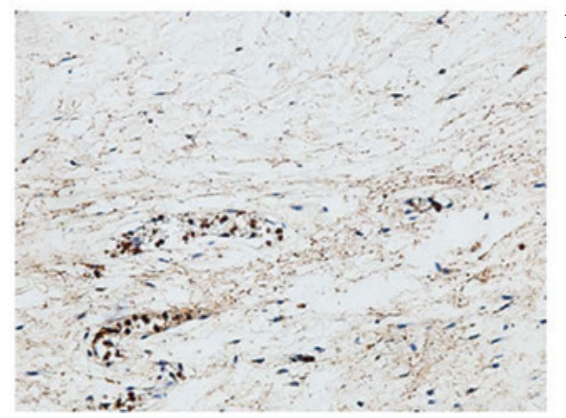

B

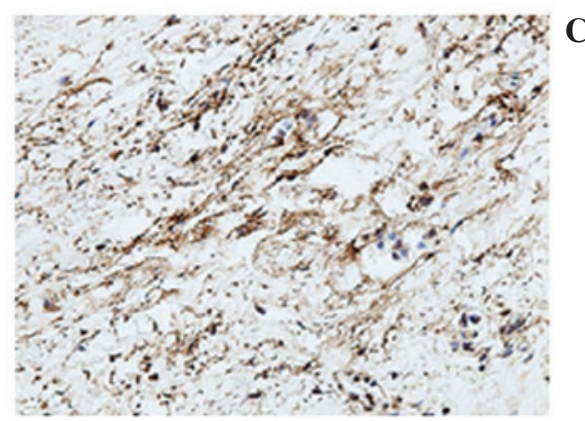

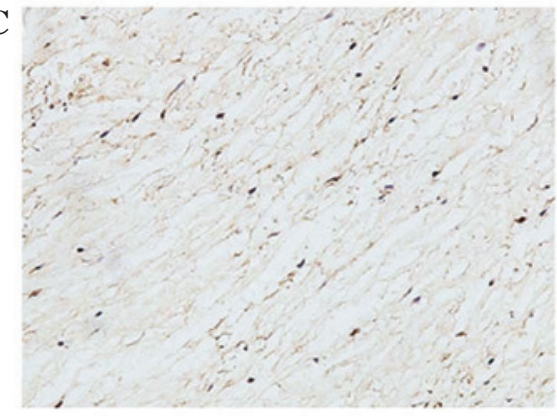

Figure 6. Immunohistochemical staining for matrix metalloproteinase-13 (magnification, $x 200$ ) in specimens of the (A) CON, (B) ORC and (C) CNT groups . Staining intensity was greater in every specimen of the ORC group compared with every specimen of the CON and CNT groups. CON, non-operated control; ORC, operated contracture; CNT, contracture-treatment. 
A

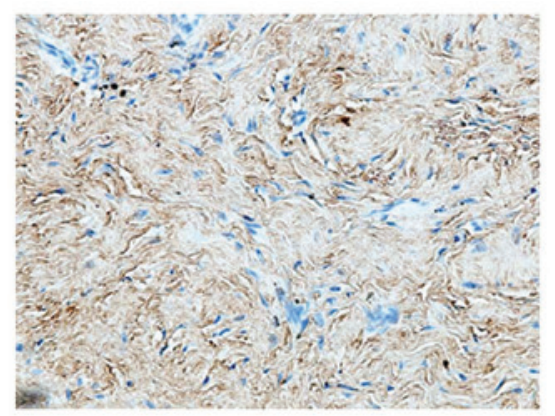

B

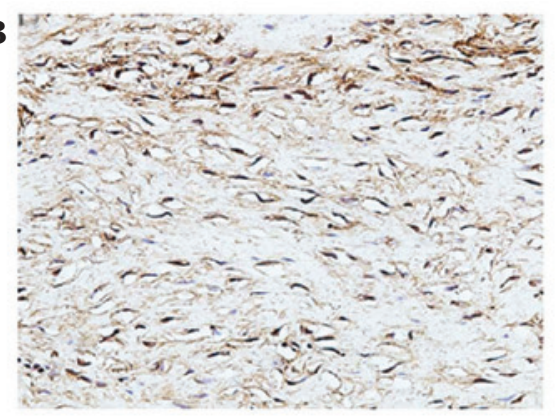

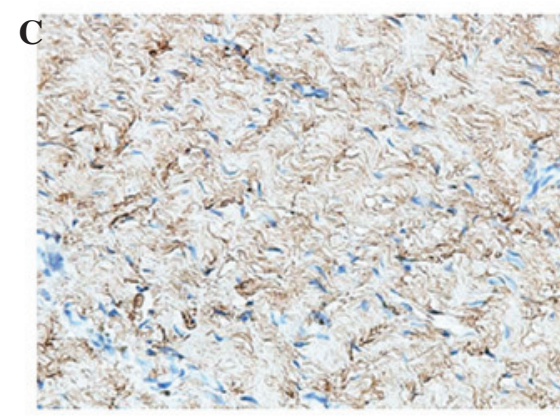

Figure 7. Immunohistochemical staining for collagen III (magnification, x200) in specimens of the (A) CON, (B) ORC and (C) CNT groups. Staining intensity was decreased in every specimen of the ORC group compared with every specimen of the CON and CNT groups. CON, non-operated control; ORC, operated contracture; CNT, contracture-treatment.

A

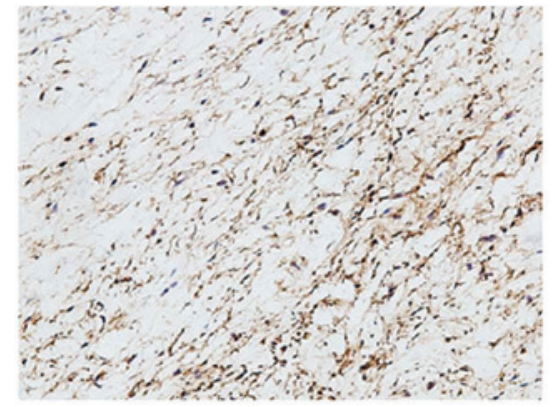

B

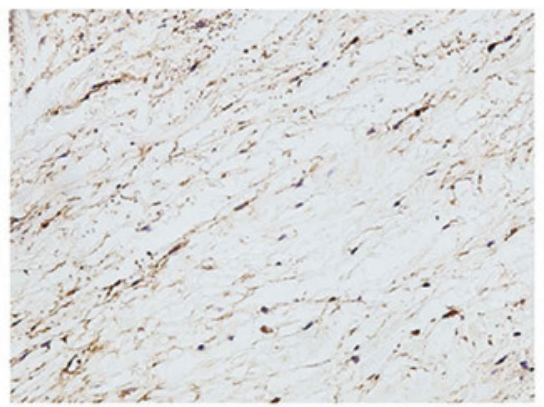

C

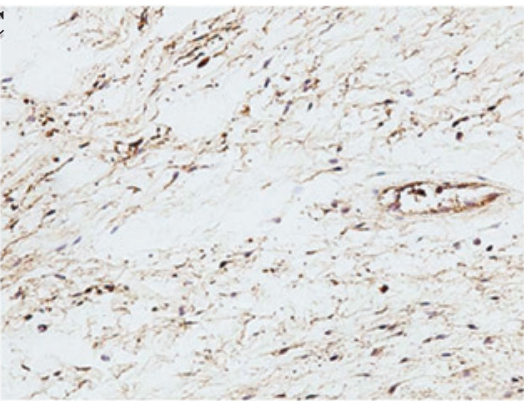

Figure 8. Immunohistochemical staining for tissue inhibitor of metalloproteinase-13 (magnification, x200) in specimens of the (A) CON, (B) ORC and (C) CNT groups. Staining intensity was decreased in every specimen of the ORC group compared with every specimen of the CON and CNT groups. CON, non-operated control; ORC, operated contracture; CNT, contracture-treatment.

\section{Discussion}

A feature of the post-traumatic joint contracture model used in the present study is irreversible loss of joint motion, irrespective of the period of remobilization (8). By contrast, other animal models of post-traumatic immobilization have revealed reversible joint motion loss following periods of remobilization equal to the duration of initial immobilization (9-11), bringing into question the unique pathological changes occurring following trauma that render the joint susceptible to irreversible joint contractures. Based on previous studies in animals and humans, joint capsule fibrosis observed in post-traumatic joint contractures may account for this irreversible loss of joint motion; myofibroblasts are considered to be the cell type predominantly involved in the development of joint capsule fibrosis (12-14), and elbow extension-flexion motion is inversely proportional to the number of myofibroblasts present within the capsule of the elbow joint (15). A previous study using the current model also supports this hypothesis, as the joint capsules from rats of the ORC group were densely filled with myofibroblasts (7). In addition, treatment of the CNT group with LV-mediated ERK2 siRNA significantly reduced (or prevented) the number of myofibroblasts within the joint capsule following trauma-induced joint contracture (7). However, this previous study did not investigate extracellular matrix turnover of the fibrotic joint capsule, leading to its evaluation in the current study.

The joint capsule is composed mainly of collagen fibers and the normal joint capsule is compliant, well-organized and thin. The present study evaluated the morphological characteristics and the expression of specific types of collagen in the fibrotic capsule in order to reveal the structural and biochemical changes of the capsule that may lead to motion limitation of the joint following traumatic injury. Collagen content is a key factor that determines the mechanical strength of healing tissue (16-18). The relative proportions of collagen I and III as a proportion of the total collagen are another determinant of the mechanical properties of a tissue, with a higher proportion of collagen III hypothesized to reduce the strength of the fibrotic joint capsule by decreasing fiber diameter (16-18). One previous study demonstrated that the expression levels of collagen I and III proteins, which are major constituents of contracted tissue, were also increased in fibrotic joint capsules (19). Concordantly, the present study revealed that staining of total collagen and collagen I was more intense in the contracted capsules than in the control capsules. In contrast to total collagen and collagen I, staining for collagen III was more intense in the control capsules, which is in agreement with the results of the study by Matsumoto et al (20). These results demonstrate a mechanism of contracture tissue formation that differs from that of wound healing, with increased levels of collagen type III $(21,22)$. Similar to total collagen and collagen I, the levels of MMP-13 and MMP-1 were increased in the capsule of the ORC group compared with the CON group in the present study. By contrast, TIMP-13 was decreased in the control capsules compared with the contracted capsules. This high matrix turnover of joint capsule in the fibrotic joint capsule has also been reported in previous studies $(21,22)$. An 
increase of pERK2 in rats with trauma-induced joint capsule fibrosis was observed in the present study, which may be responsible for these pathological alterations. Furthermore, LV-mediated ERK2 siRNA was responsible for pathological alterations in the joint capsular tissue of the CNT group by downregulating pERK2 levels, which may reflect the important role of pERK2 in these pathological alterations.

The results of the present study demonstrated that high extracellular matrix turnover has an important role in the pathogenesis of the fibrotic joint capsule, and that the phosphorylation of ERK2 is a key factor in reducing joint capsule fibrosis by changing extracellular matrix turnover.

\section{Acknowledgements}

The present study was supported by the Chinese National Natural Science Foundation (grant no. GSCX0818005).

\section{References}

1. Lindenhovius AL and Jupiter JB: The posttraumatic stiff elbow: A review of the literature. J Hand Surg Am 32: 1605-1623, 2007.

2. Gallay SH, Richards RR and O'Driscoll SW: Intraarticular capacity and compliance of stiff and normal elbows. Arthroscopy 9: 9-13, 1993.

3. Cohen MS, Schimmel DR, Masuda K, Hastings H 2nd and Muehleman C: Structural and biochemical evaluation of the elbow capsule after trauma. J Shoulder Elbow Surg 16: 484-490, 2007.

4. Hildebrand KA, Zhang M, Germscheid NM, Wang C and Hart DA: Cellular, matrix, and growth factor components of the joint capsule are modified early in the process of posttraumatic contracture formation in a rabbit model. Acta Orthop 79: 116-125, 2008.

5. Monument MJ, Hart DA, Befus AD, Salo PT, Zhang M and Hildebrand KA: The mast cell stabilizer ketotifen reduces joint capsule fibrosis in a rabbit model of post-traumatic joint contractures. Inflamm Res 61: 285-292, 2012.

6. Monument MJ, Hart DA, Befus AD, Salo PT, Zhang M and Hildebrand KA: The mast cell stabilizer ketotifen fumarate lessens contracture severity and myofibroblast hyperplasia: A study of a rabbit model of posttraumatic joint contractures. J Bone Joint Surg Am 92: 1468-1477, 2010.

7. Li F, Liu S and Fan C: Lentivirus-mediated ERK2 siRNA reduces joint capsule fibrosis in a rat model of post-traumatic joint contracture. Int J Mol Sci 14: 20833-20844, 2013.
8. Hildebrand KA, Zhang M and Hart DA: Joint capsule matrix turnover in a rabbit model of chronic joint contractures: Correlation with human contractures. J Orthop Res 24: 1036-1043, 2006

9. Akeson WH, Woo SL, Amiel D and Doty DH: Rapid recovery from contracture in rabbit hindlimb. A correlative biomechanical and biochemical study. Clin Orthop Relat Res: 359-365, 1977.

10. Finsterbush A and Friedman B: Reversibility of joint changes produced by immobilization in rabbits. Clin Orthop Relat Res: 290-298, 1975.

11. Schollmeier G, Sarkar K, Fukuhara K and Uhthoff HK: Structural and functional changes in the canine shoulder after cessation of immobilization. Clin Orthop Relat Res: 310-315, 1996.

12. Hildebrand KA, Zhang M, van Snellenberg W, King GJ and Hart DA: Myofibroblast numbers are elevated in human elbow capsules after trauma. Clin Orthop Relat Res: 189-197, 2004.

13. Abdel MP, Morrey ME, Barlow JD, Kreofsky CR, An KN, Steinmann SP, Morrey BF and Sanchez-Sotelo J: Myofibroblast cells are preferentially expressed early in a rabbit model of joint contracture. J Orthop Res 30: 713-719, 2012.

14. Hildebrand KA, Sutherland C and Zhang M: Rabbit knee model of post-traumatic joint contractures: The long-term natural history of motion loss and myofibroblasts. J Orthop Res 22: 313-320, 2004.

15. Germscheid NM and Hildebrand KA: Regional variation is present in elbow capsules after injury. Clin Orthop Relat Res 450: 219-224, 2006.

16. Fukui N, Tashiro T, Hiraoka $\mathrm{H}$, Oda $\mathrm{H}$ and Nakamura $\mathrm{K}$ : Adhesion formation can be reduced by the suppression of transforming growth factor-betal activity. J Orthop Res 18: 212-219, 2000.

17. Fukui N, Nakajima K, Tashiro T, Oda $H$ and Nakamura K: Neutralization of fibroblast growth factor-2 reduces intraarticular adhesions. Clin Orthop Relat Res: 250-258, 2001.

18. Fukui N, Fukuda A, Kojima K, Nakajima K, Oda H and Nakamura K: Suppression of fibrous adhesion by proteoglycan decorin. J Orthop Res 19: 456-462, 2001.

19. Hildebrand KA, Zhang M and Hart DA: High rate of joint capsule matrix turnover in chronic human elbow contractures. Clin Orthop Relat Res 439: 228-234, 2005.

20. Matsumoto F, Trudel G and Uhthoff HK: High collagen type I and low collagen type III levels in knee joint contracture: An immunohistochemical study with histological correlate. Acta Orthop Scand 73: 335-343, 2002.

21. Robins SP, Milne G, Duncan A, Davies C, Butt R, Greiling D and James IT: Increased skin collagen extractability and proportions of collagen type III are not normalized after 6 months healing of human excisional wounds. J Invest Dermatol 121: 267-272, 2003.

22. Sculean A, Junker R, Donos N, Berakdar M, Brecx M and Dünker N: Immunohistochemical evaluation of matrix molecules associated with wound healing following regenerative periodontal treatment in monkeys. Clin Oral Investig 6: 175-182, 2002 . 